\title{
Improved sperm cryosurvival in diluents containing amides versus glycerol in the Przewalski's horse (Equus ferus przewalskii) ${ }^{\text {is }}$
}

\author{
Budhan S. Pukazhenthi ${ }^{\mathrm{a}, *}$, Aime Johnson ${ }^{\mathrm{b}}$, H. David Guthrie ${ }^{\mathrm{c}}$, Nucharin Songsasen ${ }^{\mathrm{a}}$, Luis R. Padilla ${ }^{\mathrm{a}, 1}$, \\ Barbara A. Wolfe ${ }^{\mathrm{d}}$, Marco Coutinho da Silva ${ }^{\mathrm{e}}$, Marco A. Alvarenga ${ }^{\mathrm{f}}$, David E. Wildt ${ }^{\mathrm{a}}$ \\ a Smithsonian Conservation Biology Institute, National Zoological Park, Front Royal, VA 22630, United States \\ ${ }^{\mathrm{b}}$ Department of Clinical Sciences, College of Veterinary Medicine, Auburn University, Auburn, AL 36849, United States \\ ${ }^{\mathrm{c}}$ Animal Biosciences and Biotechnology Laboratory, Agricultural Research Service, U.S. Department of Agriculture, Beltsville, MD 20705, United States \\ ${ }^{\mathrm{d}}$ The Wilds, Cumberland, $\mathrm{OH}$ 43732, United States \\ ${ }^{\mathrm{e}}$ Department of Veterinary Clinical Sciences, College of Veterinary Medicine, The Ohio State University, Columbus, OH 43210, United States \\ ${ }^{\mathrm{f}}$ Department of Animal Reproduction and Veterinary Radiology, School of Veterinary Medicine, São Paulo State University, UNESP, Botucatu, São Paulo, Brazil
}

\section{A R T I C L E I N F O}

\section{Article history:}

Received 24 November 2013

Accepted 27 January 2014

Available online 6 February 2014

\section{Keywords:}

Equid

Spermatozoa

Semen

Cryoprotectant

Freezing

Mitochondria

\begin{abstract}
A B S T R A C $T$
Two studies were conducted to understand sperm cryosensitivity in an endangered equid, the Przewalski's horse (Equus ferus przewalski), while testing the cryoprotectant ability of formamides. The first assessed the toxicity of permeating cryoprotectants (glycerol, methylformamide [MF] and dimethylformamide [DMF]) to Przewalski's horse spermatozoa during liquid storage at $4{ }^{\circ} \mathrm{C}$. The second examined the comparative influence of three diluents (with or without formamides) on cryosurvival of sperm from the Przewalski's versus domestic horse. When Przewalski's horse spermatozoa were incubated at $4{ }^{\circ} \mathrm{C}$ in INRA 96 with differing concentrations of glycerol, MF or DMF or a combination of these amides, cells tolerated all but the highest concentration $(10 \% \mathrm{v} / \mathrm{v})$ of MF alone or in combination with DMF, both of which decreased $(P<0.05)$ motility traits. There was no effect of cryoprotectants on sperm acrosomal integrity. In the cryosurvival study, average sperm motility and proportion of cells with intact acrosomes in fresh ejaculates were similar $(P>0.05)$ between the Przewalski's $(67 \%, 84 \%$, respectively) and domestic (66\%, $76 \%$ ) horse donors. Sperm from both species were diluted in lactose-EDTA-glycerol (EQ), Botu-Crio (BOTU; a proprietary product containing glycerol and MF) or SM (INRA 96 plus $2 \%$ [v/v] egg yolk and 2.5\% [v/v] MF and DMF) and then frozen over liquid nitrogen vapor. After thawing, the highest values recovered for total and progressive sperm motility, acrosomal integrity and mitochondrial membrane potential were $42.4 \%, 21.8 \%, 88.7 \%$ and $25.4 \mathrm{CN}$ (CN = mean JC-1 fluorescence intensity/cell on a channel number scale), respectively, in the Przewalski's and $49.3 \%, 24.6 \%, 88.9 \%$ and $25.8 \mathrm{CN}$, respectively, in the domestic horse. Although sperm progressive motility and acrosome integrity did not differ $(P>0.05)$ among treatments across species, mitochondrial membrane potential was higher $(P<0.05)$ in both species using EQ compared to BOTU or SM media. Additionally, Przewalski's stallion sperm expressed higher $(P<0.05)$ post-thaw total motility in BOTU and SM compared to EQ, whereas there were no differences among freezing diluents in the domestic horse. In summary, Przewalski's stallion sperm benefit from exposure to either MF or DMF as an alternative cryoprotectant to glycerol. Overt sperm quality appears similar between the Przewalski's and domestic horse, although the total motility of cells from the former appears more sensitive to certain freezing diluents. Nonetheless, post-thaw motility and acrosomal integrity values for Przewalski's horse spermatozoa mimic findings in the domestic horse in the presence of INRA 96 supplemented with $2 \%(\mathrm{v} / \mathrm{v})$ egg yolk and a combined $2.5 \%$ concentration of MF and DMF.
\end{abstract}

Published by Elsevier Inc.

\footnotetext{
Statement of funding: This work was supported by the Sichel Endowment.

* Corresponding author. Fax: +1 5406356506.

E-mail addresses: pukazhenthib@si.edu (B.S. Pukazhenthi), akj0001@auburn.edu (A. Johnson), dave.guthrie@ars.usda.gov (H.D. Guthrie), songsasenn@si.edu (N. Songsasen), padilla@stlzoo.org (L.R. Padilla), Barbara.wolfe@cvm.osu.edu (B.A. Wolfe), Marco.CoutinhoDaSilva@cvm.osu.edu (M. Coutinho da Silva), malvarenga@fmvz.unesp.br (M.A. Alvarenga),wildtd@si.edu (D.E. Wildt).

${ }^{1}$ Current address: Saint Louis Zoo, One Government Drive, St. Louis, MO 63110, United States.
}

\section{Introduction}

The Przewalski's horse (Equus ferus przewalskii) is listed as endangered on the IUCN Red List [23]. Until the late 18th century, this species ranged from Germany and the Russian Steppes east to Kazakhstan, Mongolia and northern China. Evolutionarily, the Przewalski's horse is considered to be the last of the 'wild' horses, 
diverging from the domestic horse lineage 38,000-72,000 years ago [43]. The remaining wild Przewalski's horses were extirpated circa 1969, but reintroductions of animals produced from captive breeding have been ongoing since the 1990s. The latter efforts, occurring in Mongolia, China, Kazakhstan and Ukraine, have re-established a few small Przewalski's horse populations in the wild, but with a total number of $<400$ individuals. These herds are not self-sustaining and continue to be threatened by extreme local weather, disease and hybridization (with domestic horses). By contrast, the population of Przewalski's horses living in ex situ collections is nearly 1800 specimens distributed among 165 institutions in mostly North American and European zoos [63].

The modern Przewalski's horse population is derived from only 14 founders and, because of this extremely narrow lineage, one of the highest priorities is to retain all remaining gene diversity to ensure health, reproductive competence and species integrity [11]. This requires substantial resource commitment by holding institutions to transport genetically appropriate individuals to create breeding pairs that will produce the highest quality, heterozygous offspring. Besides the time and expense, moving large-sized, wild horses is risky and dangerous, both to animals and caretakers. The use of artificial insemination with cryopreserved sperm could facilitate moving and using germplasm between geographicallydisparate locations rather than entire animals [44,59]. While generally effective in domestic horse reproduction, artificial insemination with thawed sperm has only recently been successful in a wild equid. A milestone birth recently was achieved by our laboratories, specifically a Persian onager (Equus hemionus onager) foal [48].

Therefore, although we now know that deep-frozen, wild equid sperm can be biologically competent, large-scale application requires refinement in both the technologies and fundamental reproductive knowledge about the species, including gamete sensitivity to cryo-agents [44]. There is substantial available information on the seminal traits of Przewalski's horse electroejaculates $[8,53]$ as well as ovarian function during estrous cyclicity and pregnancy as determined by monitoring urinary estrogen conjugates [38]. Most recently, Collins et al. [9] have demonstrated that Przewalski's mares exhibit an $\sim 25$ day estrous cycle, and follicles are ovulated after reaching $\sim 40 \mathrm{~mm}$ diameter, all of which demonstrate some physiological similarities to the domestic horse. Beyond these findings, there have been no studies on the cryobiological properties of Przewalski's stallion spermatozoa, essential information for developing practical processing, banking and artificial insemination. In contrast, there is substantial knowledge about sperm cryosensitivity in the domestic horse $[2,5,15$, $20,28,29,49]$. For example, it is well-established that spermatozoa from different domestic stallions vary significantly in cryosensitivity, and that $<20 \%$ of males produce cells that express adequate post-thaw survival $[28,40,56]$. Although glycerol is used almost universally for cryopreserving stallion spermatozoa, other cryoprotectants, including methyl formamide (MF) or dimethyl formamide (DMF), have been shown to yield superior results $[1,52]$. Most domestic horse sperm cryopreservation protocols yield post-thaw motilities of $\sim 50 \%$ in stallions classified as 'good freezers', but single inseminates routinely result in $<40 \%$ of mares producing term pregnancies $[1,47]$.

The major challenge to cryopreserving spermatozoa from any species, including equids, is minimizing the toxic impact of cryoprotectants while maximizing water removal and limiting osmotic shock effects to the cell $[5,15,36]$. While glycerol remains the most common cryoprotectant for spermatozoa, there are detriments posed by its use, including membrane damage via an osmotic effect and disruption of the actin cytoskeleton [34]. For this reason, alternative cryo-agents have been examined recently, including MF and DMF [1,52]. Molecularly, these amides are smaller than glycerol, thereby facilitating and accelerating sperm membrane permeation.
Thus, the cell is exposed to the cryoprotectant for a shorter interval pre-cooling which decreases toxic effect potential. There is evidence in the domestic horse that such amides protect both sperm structure and function during freezing and thawing [1,52]. These compounds also may benefit those select stallions that produce spermatozoa that typically freeze 'poorly' for unknown reasons [1]. Finally, domestic horse sperm cooled and frozen in cryodiluents containing amides have been used to produce high rates $(>40 \%)$ of pregnancy success after artificial insemination [1,39].

The studies described here had two purposes. First, we were interested in determining the influence of permeating cryoprotectant concentration, including tolerance of Przewalski's horse spermatozoa to these formamides, specifically MF and DMF already proven advantageous for the domestic horse [1,52]. The second objective was to compare two conventional equine cryo-diluents (EQ which is comprised of lactose-EDTA plus egg yolk plus glycerol; and Botu-Crio ${ }^{\circledR}$, a publically undefined commercial product) to our 'test medium' (INRA 96 containing 2\% egg yolk and both MF and DMF) on post-thaw sperm survival. Lastly, to examine species specificity, we simultaneously compared treatments and sperm post-thaw traits in the Przewalski's versus domestic stallion. To best understand the influence of treatment on post-thaw sperm function, we relied on a multi-trait assessment, including traditional metrics (sperm total and progressive motility, acrosomal integrity) as well as mitochondrial membrane potential, a measure of mitochondrial ATP production [16]. Mitochondrial membrane potential (MMP) is known to be positively correlated with sperm motility $[16,32]$.

\section{Materials and methods}

\section{Animals}

All animal procedures were reviewed and approved by the Animal Care and Use Committees of the Smithsonian Conservation Biology Institute, the Wilds and Auburn University. Przewalski's stallions were housed individually with free access to indoor and outdoor enclosures at the Smithsonian Conservation Biology Institute $\left(78.17^{\circ} \mathrm{N}, 38.88^{\circ} \mathrm{W} ; n=7\right.$ stallions; age, $4-24$ years of age) or in a bachelor group on a 40 hectare pasture at the Wilds $\left(81.75^{\circ} \mathrm{N}\right.$ $38.82^{\circ} \mathrm{W} ; n=3$ stallions; age, $5-17$ years). These males were fed $0.5-1 \mathrm{~kg}$ of Zeigler ADF-25 herbivore diet (Zeigler Bros, Inc. Gardners, PA) supplemented with $2-4$ h of pasture access per day, orchard grass hay and ad libitum availability of water. At SCBI, stallions were supplemented with commercial oral vitamin E supplements for 3-7 days prior to an anesthetic event, but were fasted for 12-18 h immediately before anesthetic induction. Domestic stallions ( $n=5$; 3 warmblood, 1 paint, 1 quarter horse; age, $4-20$ years) were housed individually at Auburn University $\left(32.60^{\circ} \mathrm{N}, 85.48^{\circ} \mathrm{W}\right)$ and were proven breeders that also were already trained to an artificial vagina for semen collection. These males were maintained in a free-access, outdoor-indoor paddock/barn facility and were provided a generic $12 \%$ protein pellet ration twice daily with ad libitum availability to coastal Bermuda hay and water.

\section{Semen collection and assessment}

Due to the wild, dangerous behavior of Przewalski's horses, all semen collections were by electroejaculation, as previously described $[8,22,53]$ and under a surgical plane of anesthesia. Each stallion was sedated prior to anesthesia and depth of anesthesia titrated, as needed. Pre-medication involved intramuscular administration of a combination of detomidine (Pfizer Animal Health, Florham Park, NJ; 20-30 mg per horse) and ketamine hydrochloride (ZooPharm, Windsor, CO; 200-400 mg per horse). 
When each stallion displayed significant sedation (wide stance, drooped head), anesthesia was induced by intramuscular administration of etorphine hydrochloride (ZooPharm; 3-5 mg per horse) and additional ketamine (ZooPharm; 200-400 mg per horse). Each male was assisted onto lateral recumbency, and then guaifenesin (Wedgewood Pharmacy, Swedesboro, NJ) or propofol (Abbott Pharmaceuticals, Abbott Park, IL) were administered intravenously by jugular catheter to maintain a desired plane of anesthesia. Each stallion was mechanically-ventilated using a positive pressure demand valve and $100 \%$ oxygen after endotracheal intubation.

For semen collection, a sine-wave electrostimulator (AC, $60 \mathrm{~Hz}$; P.T. Electronics, Boring, OR) and a lubricated Teflon rectal probe $(5.6 \mathrm{~cm}$ diameter; P.T. Electronics) were used to administer 80-100 total stimuli in three series (30-40 stimuli per series; $3 \mathrm{~s}$ on-off pattern) while incrementally increasing the stimulation voltage (from 2 to eventually $6 \mathrm{~V}$ ). Upon achieving anesthesia, the entire semen collection interval lasted $\sim 45 \mathrm{~min}$. Of the total of 21 semen collection attempts in Przewalski's horses, 21 (100\%) produced spermic ejaculates meeting our motility criteria (see below) to be included in the study. Six of these were produced by one individual, four from another and two other males contributed three and two ejaculates, respectively. The remaining six stallions produced one semen sample each.

Ejaculates resulting from each stimulatory series were collected in pre-warmed, sterile cups $(8 \mathrm{oz})$, the fluid measured volumetrically and $\mathrm{pH}$ determined using a colorimetric strip (EMD ColorpHast pH Strips, Fisher Scientific, Waltham, MA). Semen osmolality was determined by placing a $5 \mathrm{~mm}$ filter disc saturated with $10 \mu \mathrm{l}$ of raw ejaculate into a calibrated osmometer (Wescor Inc., Logan, UT). A $10 \mu \mathrm{l}$ aliquot of the combined raw ejaculate was fixed in $0.5 \mathrm{ml}$ of $0.3 \%$ glutaraldehyde (in phosphate buffer saline [PBS], pH 7.4, osmolality $340 \mathrm{mOsm}$ ) and stored at $4{ }^{\circ} \mathrm{C}$ until assessed for sperm morphology. This evaluation examined 100 total spermatozoa per ejaculate via phase contrast microscopy (1000×; BX40, Olympus Corporation; Center Valley, PA) for normal structure or malformation of the head, midpiece and flagellar regions as well as presence of a proximal or distal cytoplasmic droplet. A $40 \mu \mathrm{l}$ aliquot of raw ejaculate also was fixed in $0.5 \mathrm{ml}$ of $4 \%$ paraformaldehyde in PBS and stored at $4{ }^{\circ} \mathrm{C}$ until assessed for acrosomal integrity using Coomassie blue (see below for more detail) [25].

For sperm motility assessment, raw ejaculate from each series was immediately diluted $1: 2$ or $1: 3$ with warm INRA 96 (INRA 96, IMV Technologies, Maple Grove, MN), subjectively evaluated $(200 \times)$ for estimated percentage of motile sperm (TM) and the proportion moving progressively in a rapid, straightforward direction (PM). Ejaculate fractions with similar motility traits and meeting the criteria of $\geqslant 60 \% \mathrm{TM}$ and $\geqslant 50 \%$ PM were combined in a fresh collection cup and poured through an equine semen filter (Agtech Inc., Manhattan, KS). A $10 \mu \mathrm{l}$ aliquot of the pooled semen was diluted further $(1: 200)$ and evaluated for sperm concentration using a standard hemocytometer approach [21].

Domestic horses (1 ejaculate/stallion) were collected using a Missouri Model artificial vagina with an in-line gel filter (HarVet, Spring Valley, WI) off a breeding phantom [31]. All five semen samples met our minimum sperm motility criteria (described above) and were processed generally within 15 min of collection (as described above) with two exceptions: (1) cellular motility was determined using a computer assisted sperm motility analyzer (CASA, Minitube of America, SpermVision SAR); and (2) sperm concentration was assessed via a NucleoCounter SP-100 unit (Chemometec, Denmark). Both of these approaches were part of a routine diagnostic protocol for assessing horse fertility at the College of Veterinary Medicine, Auburn University.

\section{Evaluation of acrosomal integrity}

Sperm samples fixed in 4\% paraformaldehyde were centrifuged for $8 \mathrm{~min}$ at $2000 \times \mathrm{g}$ and the supernatant discarded. Pellets were washed twice with $500 \mu \mathrm{l}$ of $0.1 \mathrm{M}$ ammonium acetate $(\mathrm{pH}, 9.0)$ and the final pellet resuspended in $\sim 50 \mu \mathrm{l}$ of this same solution after which $\sim 15 \mu$ l was smeared onto two microscope slides and allowed to dry (ambient temperature). Each slide was stained with 0.22\% Coomassie stain (Coomassie Blue G-250; Thermo Fisher Scientific, Waltham, MA) in 50\% methanol, $10 \%$ glacial acetic acid and $40 \%$ deionized water for $90 \mathrm{~s}$ [25]. All slides were rinsed with deionized water, dried (ambient temperature) and permanently preserved by placing a cover slip over a drop of mounting medium (Krystalon; EM Science, Gibbstown, NJ). For each sample, 200 spermatozoa were assessed individually for acrosomal integrity using bright field microscopy at $1000 \times$ and categorized as intact, damaged or non-intact. Briefly, cells from both species with intact acrosomal membranes exhibited uniform blue staining overlying the acrosomal region. A spermatozoon lacking an intact acrosome displayed a clear (unstained) area overlying this region, whereas cells with damaged membranes expressed a patchy staining pattern.

\section{Assessment of mitochondrial membrane potential}

Mitochondrial energy status was determined in spermatozoa loaded with the probe 5,5',6,6'-tetrachloro-1,1',3,3'-tetraethylbenzimidazolylcarbocyanine iodide (JC-1) (T-3168, Molecular Probes Inc., Eugene, OR) using a procedure modified from a boar sperm study [16]. To measure mitochondrial inner membrane potential, the cryodiluent was removed from each thawed sperm suspension by diluting in INRA $96-120 \times 10^{6} \mathrm{sperm} / \mathrm{ml}$. This was followed by centrifugation $(1000 \times g)$ in a two-step discontinuous Percoll (P-1644, Aldrich, Milwaukee, WI) density gradient consisting of $35 \%$ and then $70 \%$ isotonic Percoll [17]. Each resulting sperm pellet (collected in a $12 \times 75 \mathrm{~mm}$ polypropylene tube) was washed and diluted in $1 \mathrm{ml}$ of INRA 96 containing $4 \mu \mathrm{l}$ of propidium iodide (final concentration $9.6 \mu \mathrm{M}$ ) and $4.5 \mu \mathrm{l}$ of JC-1 working solution (final concentration $2.25 \mu \mathrm{M}$ ) to an end concentration of $10 \times 10^{6}$ sperm $/ \mathrm{ml}$. The cells plus reagents were incubated for $20 \mathrm{~min}$ $\left(37^{\circ} \mathrm{C}\right)$ before analysis in a Beckman-Coulter XL-MCL flow cytometer using $488 \mathrm{~nm}$ excitation from a $15 \mathrm{~mW}$ air cooled argon laser. For each sample, 10,000 sperm events were selected by light scatter to exclude doublets and debris. Spermatozoa with energized mitochondria (high mitochondrial inner membrane potential $>80-100 \mathrm{mV}$ ) emitted red-orange fluorescence from JC-1. The mean JC-1 fluorescence intensity/cell on a channel number (CN) scale was calculated by the Beckman-Coulter XL-MCL System II Software.

\section{Study 1: Influence of cryoprotectant concentration as well as} formamides as alternative cryo-agents on Przewalski's horse sperm motility and acrosomal integrity

Four ejaculates ( $1 /$ male) were washed (as described above) and sperm pellets resuspended in INRA 96 and then subdivided into 16 aliquots of $0.5 \mathrm{ml}$ each. Four of these subsamples were treated with differing concentrations of glycerol $(0 \%, 8 \%, 16 \%$ or $32 \% ; 1: 1 \mathrm{v} / \mathrm{v})$ in a drop-wise fashion to produce a final suspension of $0 \%, 4 \%, 8 \%$ and $16 \%$ of this cryoprotectant, respectively. Another eight aliquots were diluted $1: 1(\mathrm{v} / \mathrm{v})$ with INRA 96 containing $0 \%, 5 \%, 10 \%$ or $20 \%$ methylformamide (MF) or dimethylformamide (DMF) to produce final concentrations of $0 \%, 2.5 \%, 5 \%$ or $10 \%$, respectively. The remaining four aliquots were diluted $1: 1(\mathrm{v} / \mathrm{v})$ with INRA 96 containing both $\mathrm{MF}$ and $\mathrm{DMF}(0 \%, 5 \%, 10 \%$ or $20 \%$ in equal proportions) to produce a final concentration of $0 \%, 2.5 \%, 5 \%$ or $10 \%$ each of MF combined with DMF. All tubes were cooled in a $300 \mathrm{ml}$ water 
jacket from an ambient temperature of 25 to $4{ }^{\circ} \mathrm{C}$ and maintained at this temperature for $24 \mathrm{~h}$. At 0, 4, 12 and $24 \mathrm{~h}$, subaliquots were taken for individual assessments of sperm motility, progressive motility and acrosomal integrity.

Study 2: Influence of glycerol versus combined amides in cryosurvival of Przewalski's horse versus domestic horse spermatozoa

One ejaculate from each of seven Przewalski's males and five domestic stallions were used. Briefly, semen meeting the sperm motility criteria (described above) was diluted $(1: 3 \mathrm{v} / \mathrm{v})$ in INRA 96 and then divided equally into three $15 \mathrm{ml}$ tubes that were centrifuged $(500 \times g ; 15 \mathrm{~min})$. After the supernatant was decanted, each sperm pellet was resuspended drop-wise in one of three extenders. The diluent EQ (E-Z Freezin LE, Animal Reproduction Systems, Chino, CA) was chosen as it is routinely used for cryopreserving domestic horse spermatozoa. BotuCrio ${ }^{\circledR}$ (BotuPharma, São Paulo, Brazil) was tested as this product also has been designed for horse spermatozoa and contains both glycerol and MF. Finally, the test diluent was SM (formulated in-house) containing MF and DMF to eliminate the dependence on a commercial freezing medium. Specifically, these three diluents consisted of the following: (1) EQ (20\% [v/v] egg yolk, 5.5\% [w/v] lactose, 1.5\% [w/v] glucose, $0.25 \%[\mathrm{v} / \mathrm{v}]$ sodium triethanolamine lauryl sulfate, $4 \%[\mathrm{v} / \mathrm{v}]$ glycerol); (2) Botu-Crio ${ }^{\circledR}$ (comprised of a proprietary base medium plus $20 \%$ [v/v] egg yolk, 1\% [v/v] glycerol and 4\% (v/v) MF); and (3) SM (comprised of INRA 96, 2\% [v/v] egg yolk, 2.5\% [v/v], MF and 2.5\% $[\mathrm{v} / \mathrm{v}] \mathrm{DMF}$ ). Sperm suspensions (diluted to produce a final concentration of $200 \times 10^{6}$ million sperm $/ \mathrm{ml}$ ) were packaged into $0.5 \mathrm{ml}$ labeled, plastic straws that were heat-sealed and cooled in a water jacket for $3 \mathrm{~h}$ (EQ extender) or $20 \mathrm{~min}$ (BotuCrio ${ }^{\circledR}$ or SM) at $5{ }^{\circ} \mathrm{C}$. Straws then were cryopreserved over liquid nitrogen vapor $(4 \mathrm{~cm}$ above liquid for $15 \mathrm{~min} ; \sim-70^{\circ} \mathrm{C} / \mathrm{min}$ ) and plunged into and then stored in liquid nitrogen [1].

Straws were stored frozen for at least 1 month before thawing after which each was thawed (for $\mathrm{EQ}$, at $37^{\circ} \mathrm{C}$ for $1 \mathrm{~min}$; for Botu-Crio and SM, $46{ }^{\circ} \mathrm{C}$ for $20 \mathrm{~s}$ ) and its contents emptied into a sterile Eppendorf tube and maintained at ambient temperature [1]. Thawed aliquots were evaluated immediately $(0 \mathrm{~h})$ and after a $2 \mathrm{~h}$ incubation for percent sperm TM, PM (subjective assessments), acrosomal integrity and sperm mitochondrial membrane potential using flow cytometry (as described above).

\section{Statistical analysis}

The statistical design for Study 1 was completely randomized with concentration of cryoprotectant as a fixed effect and time of cryoprotectant treatment as a repeated measure arranged in a $4 \times 4$ factorial treatment arrangement. For Study 2, the statistical design was completely randomized with horse species and freezing diluent as fixed effects and time after thawing as a repeated measure arranged in a $2 \times 3 \times 2$ factorial treatment arrangement.

Results are reported as means \pm SEM for overall ejaculate traits and for Study 1, whereas least-square means \pm SEM are presented for Study 2. Data from both studies were analyzed using the mixed model analysis of variance procedure [27] in the Statistical Analysis System software, release 9.2 (SAS Institute Inc., Cary, NC). The model diagnostics included testing for a normal distribution of error residuals and for homogeneity of treatment variance. Comparison of means was made using the DIFF option with a significance level of 0.05 .

\section{Results}

Over the course of the project we collected more spermic ejaculates $(n=21)$ from more stallions $(n=10)$ than reported previously for the Przewalski's horse. The seminal assessments revealed averages of $>30 \mathrm{ml}$ of electroejaculate comprised of $>4$ billion total sperm of which more than half were progressively motile (Table 1 ). There was an unusually high proportion of structurally abnormal spermatozoa $(>75 \%)$, most of which $(>50 \%)$ was related to cytoplasmic droplet retention.

When the subset of Przewalski's horse electroejaculates ( 1 each from 7 donors) designated for the cryopreservation (Study 2) was compared to domestic horse samples collected by an artificial vagina ( 1 each from 5 donors), there were more species similarities than differences (Table 2). All males produced ejaculates with similar seminal volume, sperm concentration and motility. The predominant species difference was related to overall proportions of sperm morphotypes, with $18 \%$ more malformations $(P<0.05)$ in Przewalski's compared to domestic horse spermatozoa. The most prevalent structurally-abnormal cell in the domestic horse ejaculate was a spermatozoon with a proximal droplet ( $17 \%$ ) (Table 2$)$. This observation held for the Przewalski's counterpart, although at nearly double the proportion $(\sim 35 \%$; $P<0.05)$. Likewise, there was a two-fold increase $(P<0.05)$ in cells with distally retained cytoplasmic droplets for the Przewalski's compared to domestic horse spermatozoa. Otherwise, there were no other species differences $(P>0.05)$, including in the incidence of intact acrosomes (both $>75 \%)$.

Study 1: Influence of cryoprotectant concentration as well as formamides as alternative cryo-agents on Przewalski's horse sperm motility and acrosomal integrity

The influence of the three permeable cryoprotectants (glycerol, MF and DMF) alone, or in combination for the two amides, on total motility of Przewalski's horse sperm in liquid storage is depicted in Fig. 1. Total motility decreased $(P<0.05) \sim 30 \%$ to $50 \%$ for the four cryoprotectants over the $24 \mathrm{~h}$ incubation interval. Glycerol (Fig. 1A) and DMF (Fig. 1C) concentrations at the tested levels had no impact $(P>0.05)$ on the decline in TM. However, a dose effect $(P<0.05)$ was found using MF alone (Fig. 1B) or in combination with DMF (Fig. 1D), with the $10 \%$ concentration reducing TM

Table 1

Fresh seminal and sperm traits in a cohort of 10 Przewalski's horse stallions (21 electroejaculates) $)^{\mathrm{a}}$.

\begin{tabular}{lc}
\hline Trait & Mean \pm SEM \\
\hline Seminal volume $(\mathrm{ml})$ & $32.2 \pm 2.9$ \\
Sperm concentration $\left(\times 10^{6} / \mathrm{ml}\right)$ & $125.8 \pm 21.9$ \\
Total sperm/ejaculate $\left(\times 10^{9}\right)$ & $4.1 \pm 0.8$ \\
Sperm total motility $(\%)$ & $67.2 \pm 1.8$ \\
Sperm progressive motility $(\%)$ & $56.8 \pm 1.6$ \\
pH & $7.8 \pm 0.1$ \\
Osmolality (mOSm) & $328.3 \pm 7.7$ \\
Normal spermatozoa (\%) & $24.4 \pm 3.5$ \\
Abnormal spermatozoa (\%) & \\
Microcephalic & $1.5 \pm 0.3$ \\
Bicephalic & $0.1 \pm 0.1$ \\
Abnormal acrosome & $6.2 \pm 1.3$ \\
Abnormal midpiece & $1.5 \pm 0.3$ \\
Coiled flagellum & $0.9 \pm 0.3$ \\
Biflagellate & $5.3 \pm 0.8$ \\
Bent midpiece with cytoplasmic droplet & $2.2 \pm 0.3$ \\
Bent midpiece without cytoplasmic droplet & $3.8 \pm 2.9$ \\
Bent flagellum with cytoplasmic droplet & $0.5 \pm 0.3$ \\
Bent flagellum without cytoplasmic droplet & $0.2 \pm 0.1$ \\
Proximal cytoplasmic droplet & $37.5 \pm 3.8$ \\
Distal cytoplasmic droplet & $14.4 \pm 2.6$ \\
Bent neck & $0.8 \pm 0.4$ \\
Spermatid & $1.3 \pm 0.3$ \\
Detached head & $1.3 \pm 0.6$ \\
Detached flagellum & $0.6 \pm 0.2$ \\
\hline Values are means \pm SEM.
\end{tabular}

a Values are means \pm SEM. 
Table 2

Fresh seminal and sperm traits in the Przewalski's horse versus domestic horse ${ }^{\mathrm{a}, \mathrm{b}}$.

\begin{tabular}{|c|c|c|}
\hline Trait & Przewalski's horse ${ }^{c}$ & Domestic horse $^{d}$ \\
\hline Seminal volume $(\mathrm{ml})$ & $33.3 \pm 3.8$ & $40.4 \pm 12.2$ \\
\hline Sperm concentration $\left(\times 10^{6} / \mathrm{ml}\right)$ & $130.6 \pm 42.5$ & $150.7 \pm 46.5$ \\
\hline Total sperm/ejaculate $\left(\times 10^{9}\right)$ & $4.1 \pm 1.3$ & $5.3 \pm 1.4$ \\
\hline Sperm total motility (\%) & $70.9 \pm 1.6^{\mathrm{e}}$ & $66.1 \pm 2.7^{e}$ \\
\hline Sperm progressive motility (\%) & $61.9 \pm 1.3^{\mathrm{e}}$ & $46.1 \pm 2.5^{\mathrm{e}}$ \\
\hline Normal spermatozoa (\%) & $31.0 \pm 5.9$ & $49.2 \pm 9.8$ \\
\hline Intact acrosome (\%) & $84.8 \pm 3.1$ & $76.3 \pm 4.2$ \\
\hline \multicolumn{3}{|l|}{ Abnormal spermatozoa (\%) } \\
\hline Macrocephalic & 0.0 & $0.2 \pm 0.2$ \\
\hline Microcephalic & $1.0 \pm 0.5$ & $0.4 \pm 0.2$ \\
\hline Bicephalic & 0.0 & $0.4 \pm 0.2$ \\
\hline Abnormal acrosome & $5.4 \pm 2.1$ & $7.8 \pm 1.6$ \\
\hline Abnormal midpiece & $1.4 \pm 0.3$ & $0.4 \pm 0.2$ \\
\hline Coiled flagellum & $0.4 \pm 0.3$ & $2.4 \pm 0.7$ \\
\hline Bent midpiece with cytoplasmic droplet & $6.0 \pm 1.0$ & $8.4 \pm 1.9$ \\
\hline Bent midpiece without cytoplasmic droplet & $3.8 \pm 1.1$ & $5.2 \pm 1.5$ \\
\hline Bent flagellum with cytoplasmic droplet & $0.1 \pm 0.1$ & $0.4 \pm 0.4$ \\
\hline Bent flagellum without cytoplasmic droplet & $0.3 \pm 0.3$ & 0.0 \\
\hline Proximal cytoplasmic droplet & $34.6 \pm 5.5^{*}$ & $17.4 \pm 9.4^{*}$ \\
\hline Distal cytoplasmic droplet & $10.1 \pm 2.7^{*}$ & $4.8 \pm 2.1^{*}$ \\
\hline Bent neck & $0.3 \pm 0.2$ & $0.8 \pm 0.4$ \\
\hline Spermatid & $1.4 \pm 0.4$ & $1.2 \pm 0.4$ \\
\hline Detached head & $4.1 \pm 2.1$ & $1.0 \pm 0.5$ \\
\hline Detached flagellum & $0.3 \pm 0.3$ & 0.0 \\
\hline
\end{tabular}

a Subset of Przewalski's horse ejaculates (from Table 1) that were used in Study 2 for comparing sperm cryosensitivity to samples from the domestic horse.

b Values are means \pm SEM.

c $n=7$ stallions, one ejaculate each.

d $n=5$ stallions, one ejaculate each.

e No statistical comparison was made due to difference in assessment methods (subjective versus CASA for the two species, respectively).

"Indicates difference $(P<0.05)$ in that metric between the species.
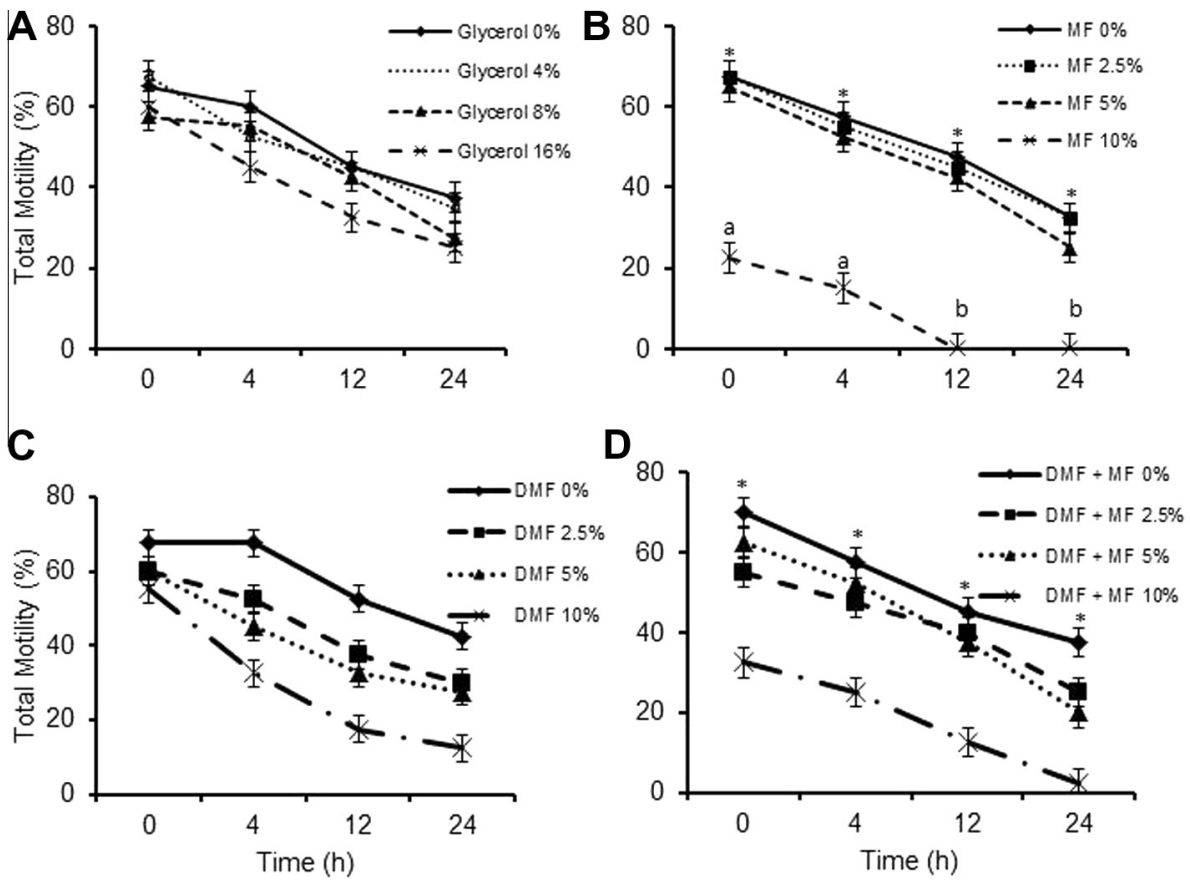

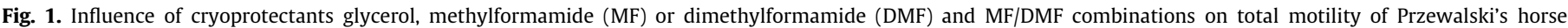

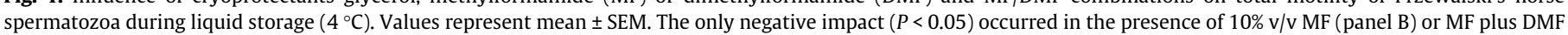

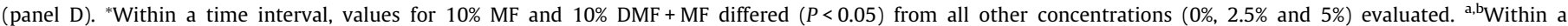
cryoprotectant concentration, values with different letters differed $(P<0.05)$ over time.

compared to other treatments. There were no differences $(P>0.05)$ in the rate of sperm motility decline among the $0 \%$ to $5 \%$ concentrations for MF (Fig. 1B) or MF plus DMF (Fig. 1D) with the pattern decrease mimicking that of the glycerol treatment (Fig. 1A). The
$10 \%$ concentration of DMF alone also appeared to shift sperm motility survival downward (Fig. 1C), but not significantly $(P>0.05)$. Another noteworthy observation was that exposure to $10 \% \mathrm{MF}$ alone (Fig. 1B) or with 10\% MF and DMF (Fig. 1D) 
immediately reduced the TM value by at least $50 \%$. Additionally, these high concentrations of amide eliminated all sperm motility by 12 (10\% MF alone; Fig. 1B) or 24 (10\% DMF and MF; Fig. 1D) h of culture.

Progressive motility (PM) profiles for Przewalski's horse spermatozoa were similar to the TM patterns. Overall, supplementation of glycerol, MF or DMF, regardless of concentration, decreased $(P<0.05)$ PM over time (Fig. 2). The profiles of decline were not influenced $(P>0.05)$ by glycerol (Fig. $2 \mathrm{~A})$ or DMF (Fig. $2 \mathrm{C}$ ) concentrations or by the $0 \%, 2.5 \%$ or $5.0 \% \mathrm{MF}$ (Fig. $2 \mathrm{~B}$ ) or these same dosages of MF plus DMF (Fig. 2D). As observed for TM, the $10 \%$ concentration of MF alone compromised $(P<0.05) \mathrm{PM}$ at culture onset and resulted in near total loss by $12 \mathrm{~h}$ of incubation (Fig. 2B).

The proportion of spermatozoa with intact acrosomes did not change $(P>0.05)$ with cryoprotectant tested or over time. Range in values over the $24 \mathrm{~h}$ culture was narrow within each cryoprotectant group (glycerol, 87-93\%; MF, 87-92\%; DMF, 85-91\%; DMF plus $\mathrm{MF}, 87-90 \%)$.

Study 2: Influence of glycerol versus combined amides in cryosurvival of Przewalski's horse versus domestic horse spermatozoa

Within each species, the percentage decrease in sperm TM from pre-freeze (fresh ejaculate for Przewalski's horse $=70.9 \%$; domestic horse $=66.1 \%$, Table 2$)$ to the first evaluation after thawing $(42.4 \%$ and $49.3 \%$, respectively) was similar $(P>0.05$; a $28.2 \%$ and $16.8 \%$ decrease, respectively). Because none of the three factor interactions was significant for the four variables analyzed (TM, PM, intact acrosomes and JC-1 fluorescence), we analyzed the two-way interactions to examine the influence of diluent (Table 3) and incubation interval (Table 4) across the two species. Disregarding specific cryodiluent, sperm TM after liquid nitrogen storage was higher $(P<0.05)$ in the domestic $(74.5 \%)$ compared to Przewalski's $(59.8 \%)$ horse, which appeared at least partially due to species var- iation in tolerance to freezing medium. For example, sperm TM was only influenced by cryodiluent $(P<0.05)$ in Przewalski's, but not domestic horse ejaculate (Table 3). Sperm TM for the Przewalski's horse was superior $(P<0.05)$ in SM compared to EQ and equivalent $(P>0.05)$ to BOTU. TM for the Przewalski's horse sperm cryopreserved in SM was equivalent $(P>0.05)$ to all three freezing diluents used for domestic horse spermatozoa. By contrast, this motility metric when using EQ was lower $(P<0.05)$ than that observed for all three cryodiluents used in the domestic horse. This species-by-cryodiluent interaction was not sustained $(P>0.05)$ for post-thaw PM or proportions of intact acrosomes (Table 3 ). In general, the proportion of sperm showing progressive movement was in the $16.1-27.6 \%$ range, whereas $88.5-89.3 \%$ of all cells were undamaged regardless of species or freezing diluent.

The average mitochondrial transmembrane potentials upon thawing ranged from 19.6-26.6 CN units (Table 3). Within each species, use of EQ resulted in the highest membrane potential scores that were different $(P<0.05)$ in both species from values measured with SM and BOTU.

The variability of thawed Przewalski's or domestic horse spermatozoa to maintain viability for $2 \mathrm{~h}$ after thawing are depicted in Table 4. While sperm TM was sustained and not different $(P>0.05)$ between the two time periods within species, TM was lower $(P<0.05)$ in the Przewalski's horse compared to the domestic counterparts. No time effects $(P>0.05)$ were observed for either species for PM or proportion of intact acrosomes, but mitochondrial membrane potential declined $(P<0.05)$ at $2 \mathrm{~h}$ in both species.

\section{Discussion}

Besides adding to our fundamental understanding of seminal traits of the Przewalski's horse, we were keen to examine the sensitivity of the spermatozoa of this endangered species to cryoprotectants, freezing, liquid nitrogen storage, thawing and
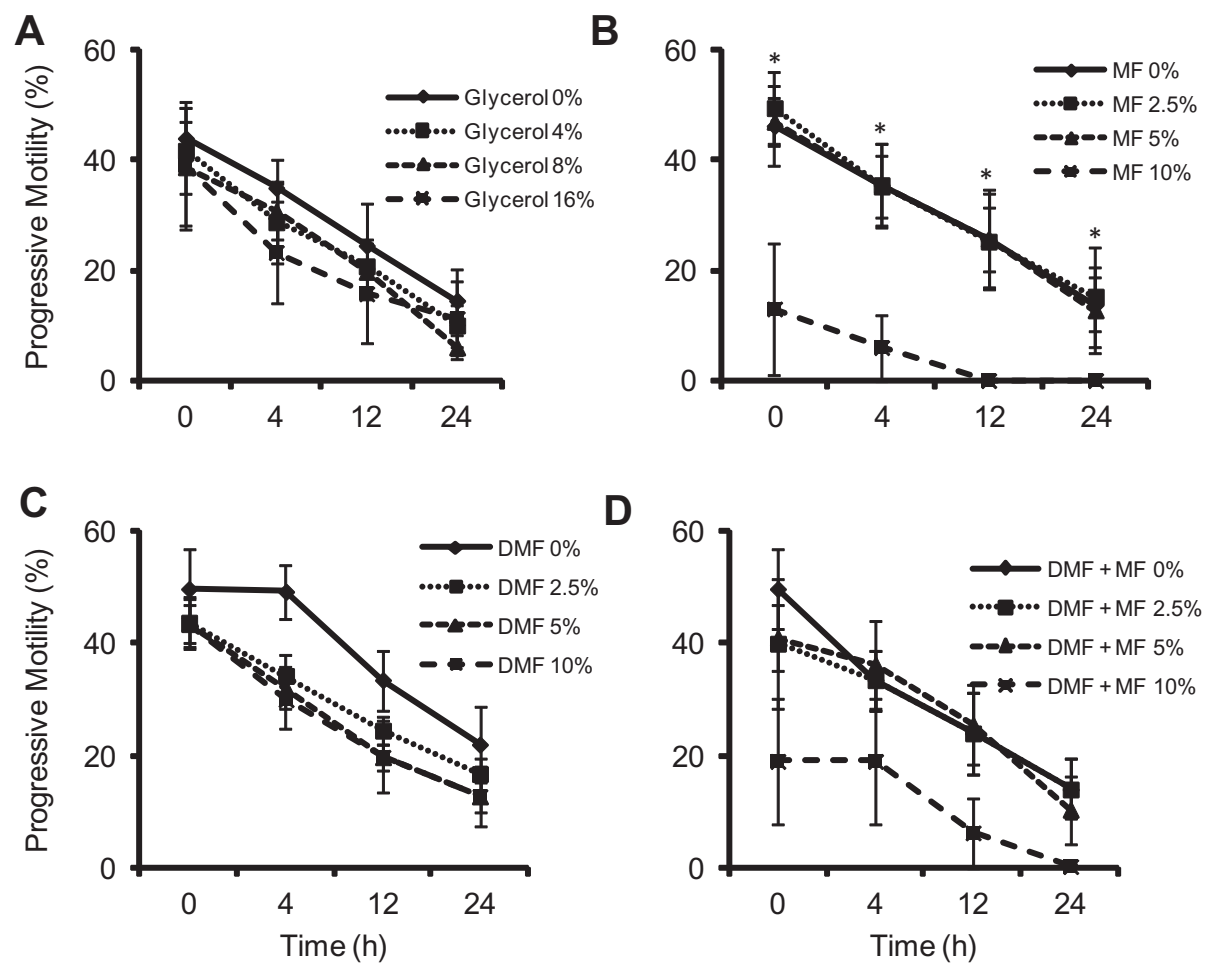

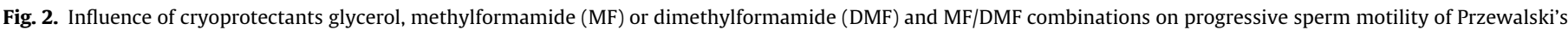

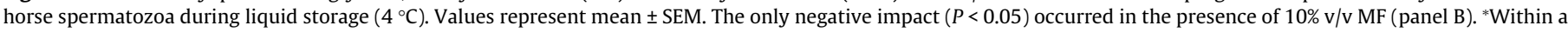
time interval, values for $10 \%$ MF differed $(P<0.05)$ from all other concentrations $(0 \%, 2.5 \%$ and $5 \%)$ evaluated. 
Table 3

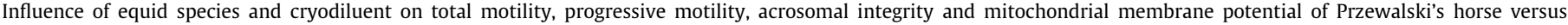
domestic horse spermatozoa (evaluations at $37^{\circ} \mathrm{C}$ ).

\begin{tabular}{|c|c|c|c|c|c|c|}
\hline & \multicolumn{3}{|c|}{ Przewalski's horse } & \multicolumn{3}{|c|}{ Domestic horse } \\
\hline & BOTU & EQ & SM & BOTU & EQ & SM \\
\hline Total motility (\%) & $42.8 \pm 3.6^{\mathrm{bc}}$ & $32.8 \pm 3.7^{c}$ & $52.1 \pm 5.4^{\mathrm{ab}}$ & $55.0 \pm 2.2^{\mathrm{a}}$ & $50.0 \pm 3.5^{\mathrm{ab}}$ & $48.0 \pm 3.0^{\mathrm{at}}$ \\
\hline Progressive motility (\%) & $23.0 \pm 2.6^{\mathrm{a}}$ & $16.1 \pm 2.8^{\mathrm{a}}$ & $27.4 \pm 5.1^{\mathrm{a}}$ & $27.6 \pm 0.6^{\mathrm{a}}$ & $24.2 \pm 2.2^{\mathrm{a}}$ & $21.4 \pm 1.8^{\mathrm{a}}$ \\
\hline Acrosome integrity (\%) & $88.5 \pm 0.3^{\mathrm{a}}$ & $89.2 \pm 0.4^{\mathrm{a}}$ & $87.9 \pm 0.4^{\mathrm{a}}$ & $88.6 \pm 0.5^{\mathrm{a}}$ & $89.3 \pm 0.2^{\mathrm{a}}$ & $88.6 \pm 0.5^{a}$ \\
\hline JC-1 fluorescence $(\mathrm{CN})$ & $21.9 \pm 1.2^{\mathrm{b}}$ & $26.6 \pm 1.2+$ & $19.6 \pm 1.2^{\mathrm{b}}$ & $22.2 \pm 2.2+$ & $24.9 \pm 2.7^{\mathrm{a}}$ & $21.7 \pm 2.6^{\mathrm{b}}$ \\
\hline
\end{tabular}

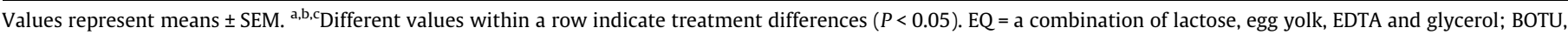
Botu-Crio = commercial proprietary product; $\mathrm{SM}=$ a combination of INRA 96, egg yolk and methyl and dimethylformamide.

Table 4

Influence of equid species and in vitro incubation time on total motility, progressive motility, acrosomal integrity and mitochondrial membrane potential of Przewalski's horse versus domestic horse spermatozoa (evaluations at $37^{\circ} \mathrm{C}$ ).

\begin{tabular}{llllll}
\hline & \multicolumn{2}{l}{ Przewalski's horse } & & \multicolumn{2}{l}{ Domestic horse } \\
\cline { 2 - 3 } \cline { 5 - 6 } \cline { 5 - 6 } & $0 \mathrm{~h}$ & $2 \mathrm{~h}$ & & $0 \mathrm{~h}$ & $2 \mathrm{~h}$ \\
\hline Total motility (\%) & $42.4 \pm 3.9^{\mathrm{a}}$ & $42.9 \pm 3.5^{\mathrm{a}}$ & & $49.3 \pm 3.1^{\mathrm{b}}$ & $52.7 \pm 2.4^{\mathrm{b}}$ \\
Progressive motility (\%) & $21.5 \pm 2.9^{\mathrm{a}}$ & $22.5 \pm 3.8^{\mathrm{a}}$ & & $24.7 \pm 1.7^{\mathrm{a}}$ & $24.1 \pm 1.1^{\mathrm{a}}$ \\
Acrosome integrity (\%) & $88.7 \pm 0.3^{\mathrm{a}}$ & $88.3 \pm 0.2^{\mathrm{a}}$ & & $88.9 \pm 0.4^{\mathrm{a}}$ & $88.8 \pm 0.3^{\mathrm{a}}$ \\
JC-1 fluorescence (CN) & $25.4 \pm 1.7^{\mathrm{a}}$ & $21.3 \pm 0.8^{\mathrm{b}}$ & & $25.8 \pm 2.4^{\mathrm{a}}$ & $20.1 \pm 2.2^{\mathrm{b}}$ \\
\hline
\end{tabular}

Values represent means \pm SEM. ${ }^{\mathrm{a}, \mathrm{b}}$ Different values within a row indicate treatment differences $(P<0.05)$

short-term incubation. We focused on methods that would have application to near-term, practical use of artificial insemination for helping maintain genetic diversity of the overall ex situ collection of this species. For this reason, we emphasized procedures and cryodiluents already known to be effective for assisted breeding of domestic horses $[1,2,29]$. Additionally, we were interested in determining the cross-species application of formamides as an alternative cryoprotectant, based on previous encouraging data produced in the common stallion [1,52]. To accomplish that objective, we conducted a comparative study that included domestic horse semen. We made three significant discoveries, including finding rather remarkable similarities in fundamental seminal traits between the two equid species. Secondly, Przewalski's horse spermatozoa maintained in liquid storage were broadly tolerant of supplementing a basic medium with either methylformamide or dimethylformamide; toxicity was observed only at the $10 \%$ concentration, with most of the detriment expressed on cellular motility. Third, it was possible to successfully freeze-thaw Przewalski's horse spermatozoa, by that meaning achieving rates of post-thaw motility, progressive motility and acrosomal integrity that mimicked values produced in counterpart cells of the domestic horse $[1,28]$. However, clearly there was some modest species-specificity, with Przewalski's horse total sperm motility being more sensitive and less viable when exposed to certain cryodiluents. Interestingly, the cryodiluent treatment producing the highest sperm survival to freeze-thawing was comprised of a combination of INRA 96 containing $2 \%(\mathrm{v} / \mathrm{v})$ egg yolk, methylformamide and dimethylformamide ( $2.5 \% \mathrm{v} / \mathrm{v}$ each).

Most domestic horse semen samples collected for routine assisted breedings are recovered using an artificial vagina [2]. On rare occasions electroejaculation has been used, but reportedly results in poorer quality samples, mostly lower sperm motility likely related to urine contamination [7]. The untamed and dangerous nature of wild equids mandates the use of electroejaculation under anesthesia and, to-date, has been applied mostly to the Persian onager $[22,48]$ and the target species of the present study, the Przewalski's horse [8,53]. Despite the potential influence of anesthetic drugs, different electronic tools and procedures, electroejaculation has been used with some effectiveness in these two species. Schook et al. [48] recently reported the recovery of high quality electroejaculates from more than half of male Persian onager donors, whereas $100 \%$ of our attempts in a large cohort of Przewalski's stallions produced more than 2.5 billion motile sperm per collection. Other general traits, including seminal volume, sperm motility and morphology also were comparable to the earlier work of Collins et al. [8] who examined fewer Przewalski's horse stallions $(n=7)$ and electroejaculates $(n=7)$.

What was particularly interesting from the present study was the similarity in quality of Przewalski's horse electroejaculates and domestic horse semen collected in an artificial vagina. When the two species were compared, the only consistently different trait was two-fold more spermatozoa with abnormal structure in the Przewalski's compared to domestic horse, almost all of which was related to retained cytoplasmic droplets, mostly situated proximally on the spermatozoon. Increased spermatozoal malformations in ejaculates can be due to seasonality or loss of genetic variation within species, populations and individuals $[12,46,55,58]$. Although substantial cause and effect data on this topic are limited for equids, Shetland ponies with high inbreeding coefficients have been shown to produce increased percentages of malformed spermatozoa [55]. Interestingly, we anecdotally calculated the inbreeding coefficients of the Przewalski's stallions used in the present study and found the overall mean value to be relatively high, $0.205 \pm 0.06$ ( $\sim 20 \%$ ). This observation and the well known fact that the modern Przewalski's horse population is derived from a small founder base [11] may have contributed to the number of structural malformations measured in our target population. It also is likely that the residual cytoplasmic droplet sperm phenotype seen in our study was related to the electroejaculation procedure itself. We suspect that these simply were immature cells being stimulated to leave the epididymis earlier than would normally occur. Heise et al. [19] have suggested that it is the admixture of spermatozoa with natural seminal fluids that promotes removal of the cytoplasmic droplet from the spermatozoon, a process that may be perturbed by electroejaculation. Interestingly, the presence of such immature cells seems unrelated to fertility in the domestic stallion [3,24,50]. Furthermore, fresh Persian onager ejaculate with traits similar to those described here for the Przewalski's horse (including the presence of $>69 \%$ immature spermatozoa) has been used to produce multiple foals by artificial insemination [48].

One of the major objectives was to determine the sensitivity of Przewalski's horse spermatozoa to cryo-agents. Historically, glycerol has been nearly the sole cryoprotectant relied upon for processing domestic horse semen for long-term storage and assisted breeding $[2,28,49]$. Its use to produce the first milestone birth of a foal from artificial insemination occurred in 1957 using thawed epididymal spermatozoa [4]. But, because glycerol can cause cell toxicity at ambient or higher temperatures and membrane disruption due to osmotic effects, alternative chemicals have been tested for horse spermatozoa, including ethylene glycol [5,52], dimethylsulfoxide [1,20,52] and propylene glycol [5]. Amides were first 
evaluated for protecting sperm from low temperature exposure in 1980 (rabbit [18]) and then later in the human [14], fowl [33,54], catfish [42], stallion [1,52], monkey [26], boar [6], koala (Phascolartos cinereus) [62], eastern gray kangaroo (Macropus giganteus) [35], donkey [57], dog [30] and tapir (Tapirus bairdii) [45]. Collectively, these findings have revealed that amides are better than, or at least similar to, glycerol in maintaining sperm viability and motility during cold storage, but with the added benefits of reducing (a) equilibration time prior to cryopreservation and (b) loss of membrane integrity from osmotic stress [1,52]. More recently, Alvarenga et al. [1] discovered that MF (5\%,v/v) and DMF (5\%, v/v) were beneficial in protecting survivability of thawed sperm from stallion donors. Furthermore, fertility trials with horse semen frozen with amides consistently yielded pregnancy rates $\geqslant 40 \%$ compared to glycerol $(<14 \%)[1]$.

Our project firstly demonstrated that total and progressive motility as well as acrosomal integrity of Przewalski's horse spermatozoa maintained at a cool liquid temperature were not adversely influenced by glycerol at any of the tested concentrations, including $5 \%$, which is used routinely in the domestic horse assisted breeding industry. Similarly processed seminal aliquots also tolerated either MF or DMF, except at the $10 \%$ concentration for MF and 5\% each of DMF and MF. Under the conditions tested, progressive motility declined precipitously in $10 \%$ MF alone without damaging acrosomal integrity. Although there was no statistical evidence for an advantage of one amide over the other for Przewalski's horse spermatozoa, there was less variation in the rate of temporal motility decline across concentrations when using MF alone $(2.5 \%$ or $5.0 \%$ ) (Fig. 1 B) or MF plus DMF ( $2.5 \%$ or $5 \%$ ) (Fig. $1 \mathrm{D})$. While the exact mechanism is unknown, we expect that the highest amide concentration was toxic and perhaps disruptive to mitochondrial function. Regardless, clearly the Przewalski's horse spermatozoon tolerated a broad range and concentration of the tested cryoprotectants.

With knowledge that Przewalski's horse spermatozoa endured $24 \mathrm{~h}$ of in vitro exposure to certain amide concentrations, cryosensitivity testing proceeded (Study 2) to examine three cryodiluents that contained glycerol or MF and/or DMF, with the latter at dosages deemed 'tolerated' in Study 1. To determine if sensitivity to freeze-thawing was conserved within the equid taxon, we compared our wild model to similarly handled spermatozoa from the domestic stallion. Germ cells from the latter withstood the described preservation and thawing protocols regardless of treatment, all resulting in $\sim 50 \%$ of the sperm being motile and $\sim 88 \%$ retaining intact acrosomes. Similar metrics were achieved in the Przewalski's horse with SM containing the MF and DMF, followed by the BOTU that contained low (1\%) glycerol and modest (4\%) MF. The least total sperm motility protection was afforded by EQ the most often used cryodiluent in the domestic horse industry. Nonetheless, although there was significant species variation, at least $32 \%$ of total sperm motility in Przewalski's horse spermatozoa was recovered with the least successful cryodiluent (EQ). A final argument that species-specificity was modest was that none of the treatments influenced progressive sperm motility or the proportion of cells with intact acrosomes.

Therefore, a significant observation of the combined findings of Studies 1 and 2 was that both Przewalski's and domestic horse spermatozoa withstood (1) cooling to $4{ }^{\circ} \mathrm{C}$ in the presence of a relatively low concentration $(2 \% \mathrm{v} / \mathrm{v})$ of egg yolk and (2) thawing after freezing over liquid nitrogen vapor, including in a skim milk, $(2 \% \mathrm{v} /$ v) egg yolk medium with only $2.5 \%$ each of MF and DMF. It appeared that the amides were at least as useful for cryoprotecting wild equid spermatozoa as glycerol, perhaps more so if focused only on total sperm motility. Glycerol is known to exert its safeguarding action via three functional hydroxyl groups each of which can accept one hydrogen from the water molecule at six different binding sites [10]. However, permeability of glycerol through the equine spermatozoal membrane is slower than that for dimethylformamide [15] which might warrant a longer equilibration time for optimal glycerol loading into such cells. Previous reports have demonstrated that protracted incubation in glycerol exposes spermatozoa to toxic influences, including membrane damage via an osmotic effect and disruption of the actin cytoskeleton [34]. By contrast, low molecular weight amides present nitrogen-containing functional groups that, in turn, bind to water's hydrogen molecules [6]. The two amides we tested are highly lipophilic (via the presence of the methyl groups) that, in turn, facilitates membrane permeability, interaction with water and reduces intracellular ice formation [6]. Particularly important is that the higher permeation capacity of amides minimizes the pre-freezing equilibration duration (at $4{ }^{\circ} \mathrm{C}$ ) for stallion spermatozoa to only 20 min, which is a substantially shorter interval than when using glycerol. This particular trait could improve semen processing efficiency in equid programs. It also would be advantageous in situations where spermatozoa need to be cryopreserved expeditiously (quickly) and under conditions that are less controlled, for example, in remote zoos or even harsh field conditions.

Our assessments of mitochondrial membrane potential at the time of sperm thawing and as a measure of cellular energy were marginally informative. Our interest in examining this metric was derived from the work of others (i.e., human [41]; bull [13]; boar [16]; dog [51]; koala [60]) who had demonstrated that freeze-thawing adversely influenced the spermatozoon's mitochondrial membrane potential. In turn, those studies had determined that sperm motility decreased markedly and was accompanied by a steep decline in ATP generation. In our investigation, mitochondrial membrane potential was lower in spermatozoa cryopreserved in BOTU and SM from both equid species compared to EQ frozen aliquots. Likewise, mitochondrial membrane potential was similar in Przewalski's and domestic horse sperm diluted and frozen in EQ. Thus, although total sperm motility was less when using the latter cryodiluent, this solution actually produced higher sperm energy metrics. It was possible that mitochondrial membrane potential was a poor indicator of sperm viability in the Przewalski's horse, especially as TM ratings were consistently at least $>40 \%$ for $2 \mathrm{~h}$ of incubation. An alternative explanation may be related to earlier work that examined energy potential in koala spermatozoa [61]. These investigators reported that thawed sperm demonstrated parallel declines in both motility and mitochondrial membrane potential (JC-1 staining). However, for certain donors, there was a disconnect between the two metrics with sperm motility maintained despite measurable mitochondrial damage. This finding suggests that there may be alternative pathways enabling ATP production for sperm motility, thereby supporting the notion that a priority should be further validation of the mitochondrial membrane potential assay as an indicator of sperm motility post-thawing in the Przewalski's horse and perhaps other equid species.

In conclusion, the Przewalski's horse is one of the seven extant, wild equid species, all of which are recognized as vulnerable to, or endangered by, extinction [37]. We discovered that spermatozoa from this species were tolerant of both cryo- (a) agents, (b) diluents and (c) processing used in the domestic horse industry. Sperm motility and acrosomal integrity levels were equivalent (either in a $4{ }^{\circ} \mathrm{C}$ liquid phase or after thawing) to what has been reported previously for the domestic horse [1] or found in the present comparative study. Particularly encouraging were the positive results achieved with the two low concentration formamides as alternatives to glycerol that, in turn, could speed the freezing-process without compromising post-thaw structure or function. We believe there are three lines of evidence that these cryopreserved sperm (regardless of cryodiluent) will be biologically competent. 
The first is that the post-thaw values we observed (that were sustained for $2 \mathrm{~h}$ in vitro) were analogous to those produced in domestic horse spermatozoa used for consistent production of foals by artificial insemination [28,29]. Secondly, Schook et al. [48] recently produced the first ever wild equid foals (in the Persian onager) after insemination of chilled or thawed spermatozoa exposed to a lactose-EDTA-egg yolk diluent containing glycerol. The cellular motility and acrosomal integrity metrics used to produce those two offspring mimicked what was produced in vitro here with the Przewalski's horse. Finally, we recently have established two term pregnancies and the birth of one surviving Przewalski's horse foal by artificial insemination using fresh sperm and inseminates with total motility, progressive motility and acrosomal integrity ratings of $\sim 65 \%, \sim 30 \%$ and $\sim 90 \%$, respectively (Pukazhenthi et al., unpublished data). The next step is to use the knowledge learned in the present study to inseminate thawed Przewalski's horse spermatozoa into conspecific mares, a project that is in progress. Meanwhile, the collective and encouraging results to-date indicate the timeliness in beginning the systematic cryobanking of genetically valuable Przewalski's horse stallions to help ensure the long-term maintenance of gene diversity and reproductive vitality of the ex situ collection.

\section{Acknowledgments}

This study was conducted under a consortium agreement of the Conservation Centers for Species Survival (C2S2), a partnership that shares unique resources to improve the biological understanding and management of endangered species, especially those requiring significant space, natural group sizes and scientific research. The authors thank: Dolores Reed and the Smithsonian Conservation Biology Institute (SCBI) staff as well as Dan Beetem and the Wilds staff for animal handling and sample collection assistance; Dr. Rachel Weiss (the Wilds) and Lisa Ware (SCBI) for veterinary assistance; Jenny Santiestevan (SCBI) for technical assistance; Glenn Welch (United States Department of Agriculture) for assistance with flow cytometric analysis; and graduate student Amy Johnson and interns Heather Richbourg, Francesca LaPiana and Katlyn Meyers for assistance with animal procedures.

\section{References}

[1] M.A. Alvarenga, F.O. Papa, F.C. Landim-Alvarenga, A.S. Medeiros, Amides as cryoprotectants for freezing stallion semen: a review, Anim. Reprod. Sci. 89 (2005) 105-113.

[2] R.P. Amann, B.W. Pickett, Principles of cryopreservation and a review of cryopreservation of stallion spermatozoa, Equine Vet. Sci. 7 (1987) 145-173.

[3] R.P. Amann, G.E. Seidel Jr., R.G. Mortimer, Fertilizing potential in vitro of semen from young beef bulls containing a high or low percentage of sperm with proximal droplet, Theriogenology 54 (2000) 1499-1515.

[4] C.A.V. Baker, J.C.C. Gandler, Pregnancy in a mare from frozen epididymal spermatozoa, Can. J. Comp. Med. Vet. Sci. 21 (1957) 47.

[5] B.A. Ball, A. Vo, Osmotic tolerance of equine spermatozoa and the effects of soluble cryoprotectants on equine sperm motility, viability and mitochondrial membrane potential, J. Androl. 22 (2001) 1061-1069.

[6] I. Bianchi, K. Calderam, E.F. Maschio, E.M. Madeira, R. da Rosa Ulguim, C.D. Corcini, D.C. Bongalhardo, E.K. Corrêa, T. Lucia Jr., J.C. Deschamps, M.N. Corrêa, Evaluation of amides and centrifugation temperature in boar semen cryopreservation, Theriogenology 69 (2008) 632-638.

[7] J.A. Cary, S. Madill, K. Farnsworth, J.T. Hayna, L. Duoos, M.L. Fahning, A comparison of electroejaculation and epididymal sperm collection techniques in stallions, Can. Vet. J. 45 (2004) 35-41.

[8] C.W. Collins, N. Songsasen, S.L. Monfort, M. Bush, B.A. Wolfe, S.B. James, D.E. Wildt, B.S. Pukazhenthi, Seminal traits in the Przewalski's horse (Equus ferus przewalskii) following electroejaculation, Anim. Reprod. Sci. 94 (2006) 46-49.

[9] C.W. Collins, N.S. Songsasen, M.M. Vick, B.A. Wolfe, R.B. Weiss, C.L. Keefer, S.L. Monfort, Abnormal reproductive patterns in Przewalski's mares are associated with a loss in gene diversity, Biol. Reprod. 86 (28) (2012) 1-10.

[10] A.M. Dalimata, J.K. Graham, Cryopreservation of rabbit spermatozoa using acetamide in combination with trehalose and methylcellulose, Theriogenology 49 (1997) 831-841.
[11] T. Fisher, D. Powell, K. Willis, Asian Wild Horse (Equus ferus przewalskii) Species Survival Plan, Association of Zoos and Aquariums, Minnesota Zoo, Mineapollis (MN), 2012. pp. 1-44.

[12] J.J. Garde, A.J. Soler, J. Cassinello, C. Crespo, A.F. Malo, G. Espeso, M. Gomendio, E.R.S. Roldan, Sperm cryopreservation in three species of endangered gazelles (Gazelle cuvieri, G. dama mhorr, and G. dorcas neglecta), Biol. Reprod. 69 (2003) 602-611.

[13] D.L. Garner, C.A. Thomas, H.W. Joerg, J.M. DeJarnette, C.E. Marshall, Fluorometric assessments of mitochondrial function and viability in cryopreserved bovine spermatozoa, Biol. Reprod. 57 (1997) 1401-1406.

[14] H.J. Glander, U.F. Haustein, The influence of the cryopreserving process on rubidium uptake by human spermatozoa, Int. J. Biol. Res. Pregnancy 3 (1982) 60-65.

[15] A.I. Glazer, S.F. Mullen, J. Liu, J.D. Benson, J.K. Critser, E.L. Squires, J.K. Graham, Osmotic tolerance limits and membrane permeability characteristics of stallion spermatozoa treated with cholesterol, Cryobiology 59 (2009) 201206.

[16] H.D. Guthrie, G.R. Welch, Determination of intracellular reactive oxygen species and high mitochondrial membrane potential in Percoll-treated viable boar sperm using fluorescence-activated flow cytometry, J. Anim. Sci. 84 (2006) 2089-2100.

[17] H.D. Guthrie, G.R. Welch, Effects of hypothermic liquid storage and cryopreservation on basal and induced membrane phospholipid disorder and acrosome exocytosis in boar spermatozoa, Reprod. Fertil. Dev. 17 (2005) 467-477.

[18] A. Hanada, H. Nagase, Cryoprotective effects of some amides on rabbit spermatozoa, J. Reprod. Fertil. 60 (1980) 247-252.

[19] A. Heise, W. Kähn, D.H. Volkmann, P.N. Thompson, D. Gerber, Influence of seminal plasma on fertility of fresh and frozen-thawed stallion epididymal spermatozoa, Anim. Reprod. Sci. 118 (2010) 48-53.

[20] N. Hoffmann, H. Oldenhof, C. Morandini, K. Rohn, H. Sieme, Optimal concentrations of cryoprotective agents for semen from stallions that are classified 'good' or 'poor' for freezing, Anim. Reprod. Sci. 125 (2011) $112-118$.

[21] J. Howard, Semen collection and analysis in carnivores, in: M. Fowler (Ed.), Zoo and Wildlife Medicine, third ed., W.B. Saunders, Philadelphia (PA), 1993, pp. 390-398.

[22] J.G. Howard, V.G. Pursel, D.E. Wildt, M. Bush, Comparison of various extenders for freeze-preservation of selective captive wild ungulates, J. Am. Vet. Med. Assoc. 179 (1981) 1157-1161.

[23] IUCN, IUCN Red List of Threatened Species. Version 2013, Downloaded 9 September 2013.

[24] A. Kavak, N. Lundeheim, M. Aidnik, S. Einarsson, Sperm morphology in Estonian and Tori breed stallions, Acta Vet. Scand. 45 (2004) 11-18.

[25] J.L. Larson, D.J. Miller, Simple histochemical stain for acrosomes on sperm from several species, Mol. Reprod. Dev. 52 (1999) 445-449.

[26] Y.H. Li, K.J. Cai, A. Kovacs, W.Z. Ji, Effects of various extenders and permeating cryoprotectants on cryopreservation of cynomolgus monkey (Macaca fascicularis) spermatozoa, J. Androl. 26 (2005) 387-395.

[27] R.C. Little, G.A. Milliken, W.W. Stroup, R.D. Wolfinger, SAS System for Mixed Models, SAS Institute Inc., Cary, NC, 1996.

[28] P.R. Loomis, The equine frozen semen industry, Anim. Reprod. Sci. 68 (2001) $191-200$.

[29] P.R. Loomis, J.K. Graham, Commercial semen freezing: individual male variation in cryosurvival and the response of stallion sperm to customized freezing protocols, Anim. Reprod. Sci. 105 (2008) 119-128.

[30] K.R. Lopes, L.L. Costa, G.L. Lima, A.L. Souza, A.R. Silva, Dimethylformamide is no better than glycerol for cryopreservation of canine semen, Theriogenology 72 (2009) 650-654

[31] C.C. Love, Semen collection techniques, Vet. Clin. North Am. Equine Pract. 8 (1992) 111-118.

[32] C.C. Love, J.A. Thompson, S.P. Brinsko, S.L. Rigby, T.L. Blancahrd, V.K. Lowry, D.D. Varner, Relationship between stallion sperm motility and viability as detected by two fluorescence staining techniques using flow cytometry, Theriogenology 60 (2003) 1127-1138.

[33] E. Lukaszewicz, DMF effects on frozen gander semen, Br. Poult. Sci. 42 (2001) 308-314.

[34] B. Marć́as Garcĺa, C. Ortega Ferrusola, IM. Aparicio, A. Miro-Moran, A. Morillo Rodriguez, J.M. Gallardo Balanos, L. Gonzalez Fernandez, C.M. Balao da Silva, H. Rodriguez Martinez, J.A. Tapia, F.J. Pena, Toxicity of glycerol for the stallion spermatozoa: effect on membrane integrity and cytoskeleton, lipid peroxidation and mitochondrial membrane potential, Theriogenology 77 (2012) 1280-1289.

[35] R. McClean, Y.P. Zee, W.V. Holt, S.D. Johnston, Cryopreservation of kangaroo spermatozoa using alternative approaches that reduce cytotoxic exposure to glycerol, Cryobiology 57 (2008) 304-307.

[36] S.A. Meyers, Spermatozoal response to osmotic stress, Anim. Reprod. Sci. 89 (2005) 57-64.

[37] P.D. Moehlman (Ed.), Equids: Zebras, Asses, and Horses: Status Survey and Conservation Action Plan, IUCN (The World Conservation Union), Gland Switzerland \& Cambridge, 2002, p. 190.

[38] S.L. Monfort, N.P. Arthur, D.E. Wildt, Monitoring ovarian function and pregnancy by evaluating excretion of urinary oestrogen conjugates in semifree-ranging Przewalski's horses (Equus przewalskii), J. Reprod. Fertil. 91 (1991) $155-164$. 
[39] G.A. Monteiro, F.O. Papa, F.S. Zahn, J.A. Dellaqua Jr., C.M. Melo, R.R.D. Maziero, B.R. Avanzi, M.A. Alvarenga, P.N. Guasti, Cryopreservation and fertility of ejaculated and epididymal stallion sperm, Anim. Reprod. Sci. 127 (2011) 197201.

[40] A. Morillo Rodriguez, C. Ortega Ferrusola, B. Macías, J.M. Morrell, H. Rodríguez Martínez, J.A. Tapia, F.J. Peña, Freezing stallion sperm with the new Cáceres extender improved post thaw sperm quality and diminishes stallion-tostallion variability, Anim. Reprod. Sci. 127 (2011) 78-83.

[41] M. O'Connell, N. McClure, S.E. Lewis, The effect of cryopreservation on sperm morphology, motility and mitochondrial function, Hum. Reprod. 17 (2002) 704-709.

[42] B. Ogier De Baulny, C. Labbe, G. Maisse, Membrane integrity mitochondria activity, ATP content and motility of European Catfish (Silurus glanis) testicular spermatozoa after freezing with different cryoprotectants, Cryobiology 39 (1999) 177-184.

[43] L. Orlando, A. Ginolhac, G. Zhang, D. Froese, A. Alberchtsen, M. Stiller, M. Schubert, E. Cappellini, B. Petersen, et al., Recalibrating Equus evolution using the genome sequence of an early Middle Pleistocene horse, Nature 499 (2013) 74-81.

[44] B.S. Pukazhenthi, D.E. Wildt, Which reproductive technologies are most relevant to studying, managing and conserving wildlife?, Reprod Fertil. Dev. 16 (2004) 33-46.

[45] B.S. Pukazhenthi, G. Della Togna, L. Padilla, D. Smith, C. Sanchez, K. Pelican, O. Sanjur, Ejaculate traits and sperm cryopreservation in the endangered Baird's tapir (Tapirus bairdii), J. Androl. 32 (2011) 260-270.

[46] M.J. Ruiz-López, N. Gañan, J.A. Godoy, A. Del Olmo, J. Garde, G. Espeso, A. Vargas, F. Martinez, E.R. Roldán, Heterozygosity-fitness correlations and inbreeding depression in two critically endangered mammals, Conserv. Biol. 26 (2012) 1121-1129.

[47] J.C. Samper, Management and fertility of mares bred with frozen semen, Anim. Reprod. Sci. 68 (2001) 219-228.

[48] M.W. Schook, D.E. Wildt, R.A. Weiss, B.A. Wolfe, K.E. Archibald, B.S. Pukazhenthi, Fundamental studies of the reproductive biology of the endangered Persian onager (Equus hemionus onager) result in first wild equid offspring from artificial insemination, Biol. Reprod. 89 (2013) 1-13.

[49] H. Sieme, R.A. Harrison, A.M. Petrunkina, Cryobiological determinants of frozen semen quality, with special reference to stallion, Anim. Reprod. Sci. 107 (2008) 276-292.

[50] L. Söderquist, L. Janson, K. Larsson, Sperm morphology and fertility in A.I. bulls, J. Vet. Med. 38 (1991) 534-543.
[51] A. Sokolowska, B.M. García, L.G. Fernández, C. Ortega-Ferrusola, J.A. Tapia, F.] Peña, Activated caspases are present in frozen-thawed canine sperm and may be related to post thaw sperm quality, Zygote 17 (2009) 297-305.

[52] E.L. Squires, S.L. Keith, K. Graham, Evaluation of alternative cryoprotectants for preserving stallion spermatozoa, Theriogenology 62 (2004) 1056-1065.

[53] J. Stover, S.W.J. Seager, E.P. Dolensk, J. Doherty, D.E. Wildt, C.C. Platz, Electroejaculation and semen evaluation in the Przewalski's horse (Equus przewalskii), Proc. Am. Assoc. Zoo. Vet. (1981) 144-145.

[54] K. Tselutin, F. Seigneurin, E. Blesbois, Comparison of cryoprotectants and methods of cryopreservation of fowl spermatozoa, Poult. Sci. 78 (1999) 586590.

55] P. van Eldik, EJ. van der Waaij, B, Ducro, A.W Kooper, T.A. Stout, B. Colenbrander, Possible negative effects of inbreeding on sperm quality in Shetland pony stallions, Theriogenology 65 (2006) 1159-1170.

[56] M. Vidament, A.M. Dupere, P. Julienne, A. Evain, P. Noue, E. Palmer, Equine frozen semen: freezability and fertility field results, Theriogenology 48 (1997) 907-917.

[57] M. Vidament, P. Vincent, F.X. Martin, M. Magistrini, E. Blesbois, Differences in ability of jennies and mares to conceive with cooled and frozen semen containing glycerol or not, Anim. Reprod. Sci. 112 (2009) 22-35.

[58] D.E. Wildt, Endangered species spermatozoa: diversity, research and conservation, in: A. Bartke (Ed.), Functions of Somatic Cells in the Testis, Springer-Verlag Inc., New York (NY), 1994, pp. 1-24.

[59] D.E. Wildt, S. Ellis, J.G. Howard, Linkage of reproductive sciences: from 'quick fix' to 'integrated' conservation, J. Reprod. Fertil. 57 (2001) 295-307.

60] Y.P. Zee, W.V. Holt, C.D. Allen, V. Nicolson, M. Burridge, A. Lisle, F.N. Carrick S.D. Johnston, Effects of cryopreservation on mitochondrial function and heterogeneity, lipid raft stability and phosphotidylserine translocation in koala (Phascolarctos cinereus) spermatozoa, Reprod. Fertil. Dev. 19 (2007) 850-860.

[61] Y.P. Zee, W.V. Holt, V. Nicolson, M. Pyne, S.D. Johnston, Individual variation in post-thaw sperm survival in a captive koala population, Cryobiology 59 (2009) 69-74.

62] Y.P. Zee, W.V. Holt, J. Gosalvez, C.D. Allen, V. Nicolson, M. Pyne, M. Burridge, F.N. Carrick, S.D. Johnston, Dimethylacetamide can be used as an alternative to glycerol for the successful cryopreservation of koala (Phascolartos cinereus) spermatozoa, Reprod. Fertil. Dev. 20 (2008) 724-733.

[63] W. Zimmerman. Przewalski's Horse Studbook (SPARKS v1.65) Equus ferus przewalskii. Compiled by: W. Zimmerman (Cologne Zoo); Data current as of 21 August 2013. 